\title{
UV-B affects the immune system and promotes nuclear abnormalities in pigmented and non-pigmented bullfrog tadpoles
}

\author{
Lilian Franco-Belussi $^{\mathrm{a}, *, 1}$, Lara Zácari Fanali ${ }^{\mathrm{b}}$, Classius De Oliveira ${ }^{\mathrm{a}}$ \\ a Department of Biology, São Paulo State University (UNESP), São José do Rio Preto, São Paulo 15054-000, Brazil \\ b Graduate Program in Animal Biology, Universidade Estadual Paulista (UNESP), São José do Rio Preto, São Paulo 15054-000, Brazil
}

\section{A R T I C L E I N F O}

\section{Keywords:}

Leukocytes

Tadpoles

Internal melanin

DNA damage

UV radiation

\begin{abstract}
A B S T R A C T
Ultra-Violet (UV) radiation is a stressor of the immune system and causes DNA damage. Leukocytes can change in response to environmental changes in anurans, making them an important biomarker of stressful situations. The initial barrier against UV in ectothermic animals is melanin-containing cells in skin and in their internal organs. Here, we tested the effects of UV exposure on immune cells and DNA integrity in pigmented and nonpigmented tadpoles of Lithobates catesbeianus. We used an inflammation model with lipopolysaccharide (LPS) of Escherichia coli to test synergic effects of UV and LPS. We tested the following hypotheses: 1) DNA damage caused by UV will be more pronounced in non-pigmented than in pigmented animals; 2) LPS increases leukocytes in both pigmented and non-pigmented animals by systemic inflammation; 3) The combined LPS and UV exposure will decrease the number of leukocytes. We found that the frequency of immune cells differed between pigmented and non-pigmented tadpoles. UV exposure increased mast cells and DNA damage in erythrocytes in both pigmented and non-pigmented tadpoles, while leukocytes decreased after UV exposure. Non-pigmented tadpoles experienced DNA damage and a lower lymphocyte count earlier than pigmented tadpoles. UV altered immune cells likely as a consequence of local and systemic inflammation. These alterations were less severe in pigmented than in non-pigmented animals. UV and LPS increased internal melanin in pigmented tadpoles, which were correlated with DNA damage and leukocytes. Here, we described for the first time the effects of UV and LPS in immune cells of pigmented and non-pigmented tadpoles. In addition, we demonstrated that internal melanin in tadpoles help in these defenses, since leukocyte responses were faster in non-pigmented animals, supporting the hypothesis that melanin is involved in the initial innate immune response.
\end{abstract}

\section{Introduction}

Ultra-Violet (UV) radiation causes behavioral and physiological changes in amphibians, contributing to their worldwide decline [1]. For example, UV radiation promotes DNA damage [1,2] by producing pyrimidine dimers that block transcription of gene and lead to mutation or cell death [3]. Another effect of UV in amphibians is disruption of the immune system [4]. Previous studies have found that exposing tadpoles in early developmental stages to ultra-violet-B (UV-B) radiation decreased their fitness as a result of the impact on the immune functions [5]. Thus, UV exposure in different life stages affects the ability of amphibians to cope with subsequent infections [5]. In addition, the combined effects of UV-B and pathogens increase the mortality of amphibian embryos more than pathogens alone [6], since UV radiation promotes systemic immunosuppression, which makes amphibians more sensitive to subsequent viral, fungal, and bacterial infections [7]. However, little is known about the systemic effects of UV. For example, how they interact with other environmental factors and defenses of amphibians against UV exposure [7]?

Hematological alterations usually reflect changes in the physiology of the organism. For example, leukocytes respond effectively to stressful situations. Therefore, these cells allow us to measure the level of damage an animal underwent [8]. Several studies have reported that the number of leukocytes change in response to different environmental variables [9-12]. Immune cells in tissues also respond to environmental change. For example, the density of liver mast cells in Physalaemus nattereri increases after $3 \mathrm{~h}$ of UV exposure [2]. Mast cells produce mediators to inflammatory process and activate neutrophils $[13,14]$ and these cells are used to indicators of inflammation in tissues [15]. In this study, mast cells were used to measure inflammation in tissues of tadpoles.

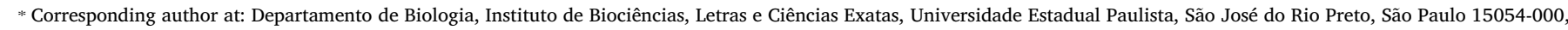
Brazil.

E-mail address: lilian.belussi@gmail.com (L. Franco-Belussi).

${ }^{1}$ Current address: Graduate program in Biotechnology and Environmental monitoring, CCTS, Federal University of São Carlos, 18052-780, Sorocaba, São Paulo, Brazil.
} 
Amphibians are susceptible to several pathogens, due to their complex life cycle. Their skin is also permeable to harmful microorganisms. For example, Escherichia coli is commonly found in environments where anurans live. The pathogenicity of this bacterium is associated with LipoPolySaccharides (LPS) in its cell wall. Specifically, the LPS activates the immune response in hosts, by stimulating mononuclear phagocytes to synthesize cytokines [16], such as the Tumor Necrosis Factor $\alpha$ (TNF- $\alpha$ ) and several Interleukins (IL-1, IL-6, and IL10 ; [17]). The LPS of $E$. coli has been commonly used in experimental models to analyse the systemic inflammatory process of rodents [18] and fish [16]. Here, we used the LPS by test synergic effects of UV and LPS.

Ectothermic animals have melanin on the surface of internal organs [19]. In the skin, melanin is the first barrier against UV [20]. Melanin also protects tissues against DNA damage by absorbing UV radiation and transforming it into heat [21]. Melanin has also an immune role in internal organs, since hydrogen peroxidases and their quinone precursors act as bactericides [22]. This is especially important for ectothermic animals, because their enzymatic reaction rates, such as enzyme-mediated repair of DNA damage induced by UV-B, decrease at low temperatures [22,23]. In addition, previous studies found that amphibian embryos died when exposed to both UV and pathogens at the same time [6]. Therefore, it is expected that the inflammation promoted by LPS would be stronger in animals exposed previously to UV. Conversely, melanin protects internal organs against UV [2]. As a result, pigmented animals are less affected due to the role of the melanin in mediating immune responses.

Here, we conducted three experiments to test if UV radiation affects immune cells and causes DNA damage on pigmented and non-pigmented tadpoles of Lithobates catesbeianus. Specifically, we tested the hypotheses that: 1) DNA damage caused by UV will be more pronounced in non-pigmented than in pigmented animals, since melanin can protect tissue against UV effects; 2) LPS increases leukocytes in both pigmented and non-pigmented animals by systemic inflammation; 3) The combined effect of LPS and UV exposure will decrease the number of leukocytes, since UV can disrupt the immune system.

\section{Methodology}

\subsection{Animal Model}

We used pigmented and non-pigmented, i.e. albine, tadpoles of the American Bullfrog (Lithobates catesbeianus; Anura: Ranidae) between stages 38 and 40 [24], supplied by Ranaville Agro Indústria Ltda, São Roque, São Paulo, Brazil. This species is bred in large scale frog farms, making it easy to obtain a homogeneous and large set of specimens, both pigmented and non-pigmented, raised under controlled conditions. Tadpoles were kept in aquaria with water equivalent to $1 \mathrm{~L}$ per individual, at room temperature $\left(27.0 \pm 0.5^{\circ} \mathrm{C}\right)$, and $12: 12$ light: dark photoperiod. Animals were fed daily for 7 days before experiments. Animal handling followed the NIH Guide for Care and Use of Laboratory Animals and procedures were approved by the Ethics and Animal Experimentation Committee of the Sao Paulo State University (CEUAIBILCE/UNESP 096/2014).

\subsection{Experiment 1: Effects of UV on Immune Cells and DNA Damage Assay}

To test the UV effects on leukocyte profile and erythrocyte abnormalities, we designed a $5 \times 2$ fully-crossed factorial experiment, in which we varied the time of exposure to UV (control group, 6, 12, 18, and $24 \mathrm{~h}$ ) and the incidence of pigmentation of tadpoles (pigmented and non-pigmented). Animals exposed for longer time to UV did not survive in a pilot study (data not shown). Each experimental group had six tadpoles (replicates). The control group consisted in animals kept under the same conditions without UV exposure. UV radiation was implemented using Philips TL $20 \mathrm{~W} / 12$ RS lamps, whose emission are $60 \%$
UVB and 40\% UVA, $<3 \%$ gamma radiation, and irradiation of $2.4 \mathrm{Wm}^{-2}$ of UVB (after $[25,26]$ ). The doses were approximately $10 \%$ of the natural UVA and UVB levels, which are average levels of summer months of southeastern Brazil [25]. Animals were kept in an incubator (EletroLab model $121 \mathrm{FC}$, with Philips TLT $20 \mathrm{~W} / 75 \mathrm{RS}$ fluorescent lamp) with controlled temperature $\left(27 \pm 0.5^{\circ} \mathrm{C}\right)$ and photoperiod (12:12 h light: dark). After exposure, animals were euthanized with a lethal dose of benzocaine diluted in water $(5 \mathrm{~g} / \mathrm{L})$, and blood and liver samples were collected. Blood was removed from the vena caudalis dorsalis of the tadpole, with syringes and heparinized needles.

\subsection{Experiment 2: Effect of LPS on Leukocytes and Hepatic Mast Cells}

To test the LPS effects on leukocyte profile and erythrocyte abnormalities, we designed a $3 \times 2$ fully-crossed factorial experiment, in which we varied the time of exposure to LPS (control group, 12 and $24 \mathrm{~h}$ ) and the incidence of pigmentation of tadpoles (pigmented and non-pigmented). Each experimental group had six tadpoles (replicates). Tadpoles were inoculated intraperitoneally with a single dose of $3 \mathrm{mg} /$ kg LPS of E. coli, Serotye 0127:B8 (Sigma, St. Louis, MO; after [16]) diluted in a sterile physiological solution with osmolality adjusted for amphibians ( $60 \%$ of mammalians). The control group consisted of pigmented and non-pigmented animals administered with a sterile physiological solution. These exposure times were chosen because during the inflammatory response, mast cells degranulate after $24 \mathrm{~h}$ [15], suggesting that the LPS are detectable during this time frame. After the end of the experiment we collected blood and liver samples.

\subsection{Experiment 3: Combined Effects of UV and LPS}

To test the combined effects of UV and LPS on leukocyte profile and erythrocyte abnormalities, we designed a $2 \times 2$ fully-crossed factorial experiment, in which we varied the time of exposure to LPS and UV (12 and $24 \mathrm{~h}$ ) and the incidence of pigmentation of tadpoles (pigmented and non-pigmented). Each experimental group had six tadpoles (replicates). Animals received an intraperitoneal single dose of LPS and exposed to $\mathrm{UV}$ following the same procedures described above.

\subsection{Histological Processing}

For hepatic mast cell analysis, the liver was fixed in Karnovsky fixative solution (0.1 M Sørensen phosphate buffer, $\mathrm{pH} 7.2$ phosphate buffer containing $5 \%$ paraformaldehyde, and $2.5 \%$ glutaraldehyde) for $24 \mathrm{~h}$ at $4{ }^{\circ} \mathrm{C}$. Subsequently, samples were washed in water, dehydrated in an alcoholic series, and embedded in historesin (Leica-historesin embedding kit). Sections of $2 \mu \mathrm{m}$ obtained in a microtome (RM 2265, Leica, Switzerland) were stained with toluidine blue and borax for mast cells detection. Sections were observed under a light microscope (Leica DM4000 B) with an image capture system (Leica DFC 280). Ten histological sections were made per animal to estimate mast cell density $\left(\mathrm{mm}^{2}\right)$.

\subsection{Leukocytes Profile and Nuclear Abnormalities of Erythorcytes}

Peripheral blood smears were fixed in methanol at $4{ }^{\circ} \mathrm{C}$ for $20 \mathrm{~min}$ and then stained with a $7.5 \%$ Giemsa solution for $15 \mathrm{~min}$. For leukocyte count, we recorded the relative proportions of each cell type per 100 leukocytes per animal under a light microscope (Leica DM4000 B). Then, we calculated the total circulating leukocytes to obtain the total amount of each cell type.

We assessed nuclear abnormalities in 1000 cells per animal under a light microscope (Leica DM4000 B), with $1000 \times$ magnification. Nuclear abnormalities in erythrocytes recorded were micronuclei, buds, binucleate erythrocytes, and anucleated cells [2]. Results were expressed as the frequency of nuclear abnormalities per 1000 cells. 

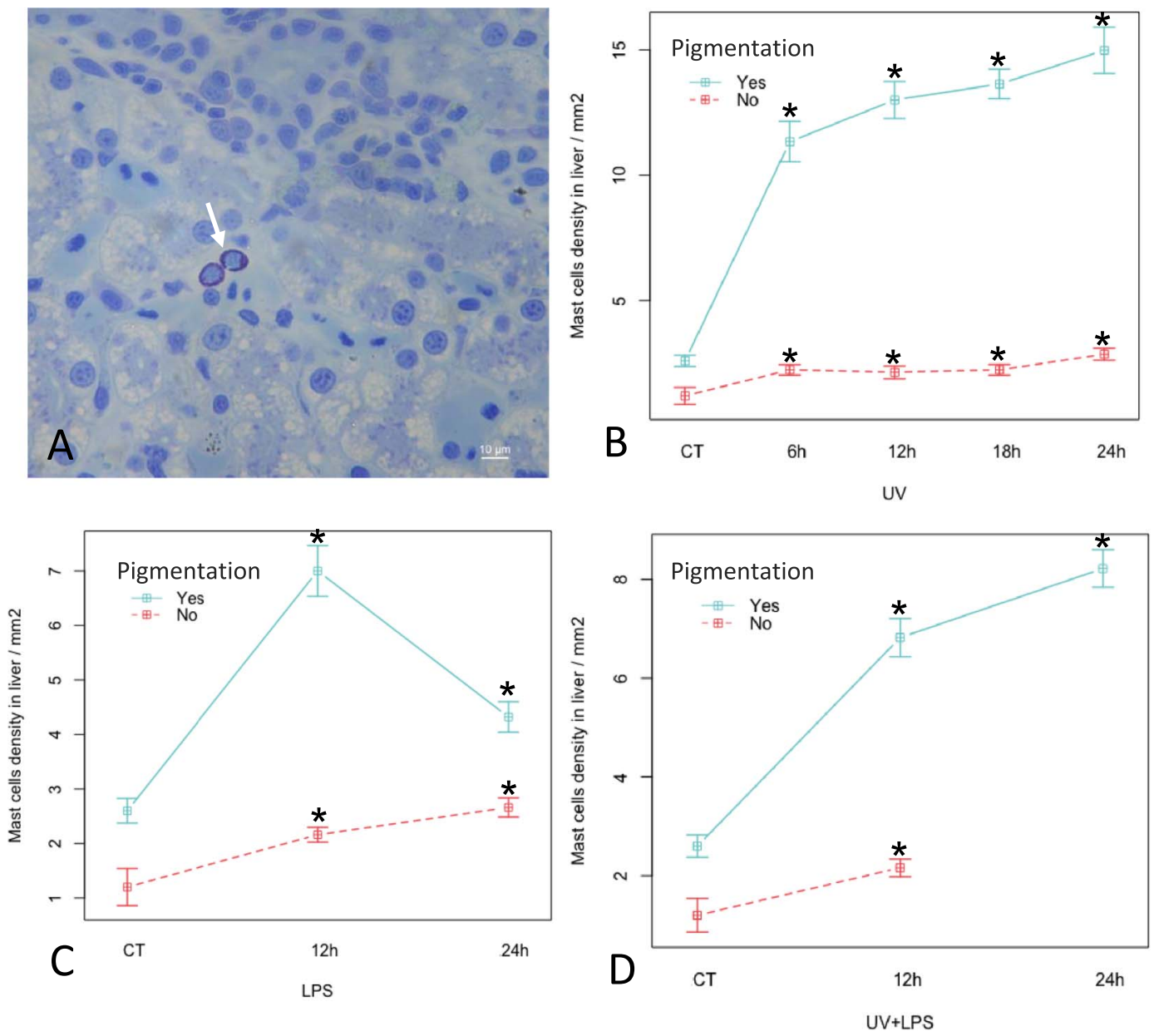

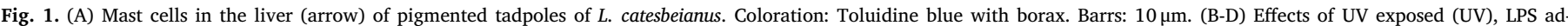

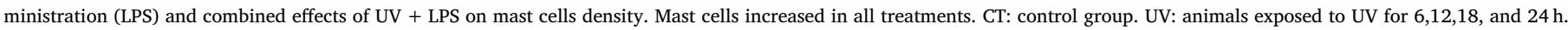

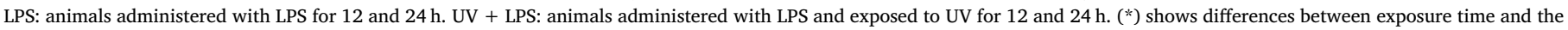
control group in the same treatment. (For interpretation of the references to color in this figure legend, the reader is referred to the web version of this article.)

\subsection{Body Darkness Analyses}

Pigmented and non-pigmented tadpoles were photographed immediately after experiments under same light conditions. To quantify body darkness, we used the delimitation tool in Photoshop ${ }^{\circledast}$ to standardize the area measured, specifically $1 \mathrm{~cm}^{2}$ of the dorsal fin and $1 \mathrm{~cm}^{2}$ between spiracle aperture and body-tail junction. These two regions were chosen to have a better sampling of what happens to the coloration in the body of the animal. We used landmarks to standardize measurements in each animal.

Images were analyzed following Franco-Belussi et al. [2] protocol for anurans using Adobe Photoshop 6.0 (Adobe Systems Inc., Mountain View, CA, USA). Briefly, we analyzed two images per animal converted into the mode CIE $L^{*} a * b *$. The format CIE $L^{*} a * b *$ [27] consists of three parameters: $\mathrm{L}^{*}=$ lightness; $\mathrm{a}^{*}=$ redness; $\mathrm{b}^{*}=$ yellowness. The mean of lightness values was measured using the histogram tool and the value $\mathrm{L}^{*}$ was inversely correlated to body darkness. Afterwards, we calculated body darkness using the following formula: $100-\left(100 \times \mathrm{L}^{*} / 255\right)$, considering the relative area of $L^{*}[2,28]$.

\subsection{Internal Melanin}

We quantified the coloration on the surface of the following regions and organs of pigmented tadpoles, following Franco-Belussi et al. [47]: heart, lungs, kidney, mesenterium, and parietal peritoneum. Briefly, the coloration on organs was divided into four categories, ranging from 0 - total absence of coloration to 3 - meaning intense pigmentation. These organs were chosen because they had some degree of pigmentation in this tadpole species at the stages analyzed.

\subsection{Statistical Analyses}

In order to model the ratio of nuclear abnormalities and leukocyte counts (lymphocytes, basophils, eosinophils, monocytes, and neutrophils), we built a Generalized Linear Model (GLM) with binomial distribution and $\log$ link function, including treatment (categorical predictor with 3 levels) and incidence of pigmentation (categorical predictor with two levels) and their interaction. We tested model assumptions using diagnostic plots in the $\mathrm{R}$ [29] package sjplot [30]. Residuals had homogeneity of variance and normal distribution.

To model mast cell density in the liver, we used a Generalized Linear Mixed-Effects Model (GLMM) with beta distribution including treatment and pigmentation as fixed effects along with their interaction. To control for the dependency among the 10 sections of the same animal (pseudoreplicates), we included animal as a random intercept in the model (categorical with 6 levels; [31,32]). Analysis was conducted in the R package gImmADMB [33]. We used the least-squares means in the $\mathrm{R}$ package lsmeans [34] to test for differences between levels of the treatment and pigmentation.

The percentage of body darkness was analyzed using a beta regression. Analysis was conducted in R package betareg [35]. To relate the categories of internal melanin in each organ or region to treatments, 
we used a G-test for goodness of fit, with Yates' correction. This test was implemented using the $\mathrm{R}$ code provided by Peter Hurd (available at www.psych.ualberta.ca/ phurd/cruft/g.test.r).

In order to test a correlation between body darkness and levels of internal melanin in organs, we used a Pearson's correlation. In addition, body darkness and internal melanin were tested with immune cells and erythrocytes nuclear abnormalities. These analyzes were conducted only for pigmented animals.

We did not observe differences between control groups of UV and LPS treatments for all responses variables. Then, we used a mean of all animals of control group. All analyses were conducted in $\mathrm{R}$ v. 3.4.0 [29].

\section{Results}

\subsection{Immune Cells in the Liver and Blood}

We found mast cells in the liver of both pigmented and non-pigmented tadpoles (Fig. 1A). The density of mast cells in the liver of pigmented animals is about twice that of non-pigmented ones (Fig. 1). The most common leukocytes in the peripheral blood of tadpoles are lymphocytes. These cells have a large nucleus and almost no cytoplasm. Neutrophils have a segmented nucleus with different configurations. Basophils, the smallest leukocytes, have the typical basophilic granules dispersed throughout the cytoplasm, sometimes overlapping the nucleus. Monocytes are the largest leukocytes, they have abundant cytoplasm bearing vacuoles, their nucleus is large, comprising about $50 \%$ of the cell volume. Eosinophils have many eosinophilic granules in their cytoplasm, with a slightly segmented and eccentric nucleus (Fig. 2).

The leukocyte profile in pigmented and non-pigmented tadpoles from the control group was different (Table 1). Pigmented animals had more lymphocytes $(78.6 \%)$ and neutrophils $(18.2 \%)$ than non-pigmented ones (lymphocytes: $70.0 \%$, neutrophils: 12.6\%). Conversely, non-pigmented animals had more eosinophils $(15.8 \%)$ than pigmented ones $(1.8 \%)$. There were no differences in monocytes and basophils.

\subsection{Effects of UV-B and LPS on Leukocytes and Hepatic Mast Cells}

UV, LPS, and UV + LPS increased mast cells in the liver (Fig. 1), but with different effects for pigmented and non-pigmented animals $(P<0.001$; Fig. 1). Pigmented animals had higher density of hepatic mast cells after $24 \mathrm{~h}$ of UV exposure, compared with non-pigmented ones (Fig. 1).

Lymphocyte decreased in all treatments $(P<0.05)$ in both pigmented and non-pigmented animals, but the decrease occurred earlier for non-pigmented than for pigmented animals $(P<0.05$; Tables 1,2 , and 3). There was an increase in neutrophils only in pigmented animals for the UV and LPS experiment after $24 \mathrm{~h}$ of exposure. Eosinophils increased only in the UV + LPS experiment in pigmented animals in two exposure times (Tables 3 ), whereas monocytes increased in all experiments for non-pigmented animals $(P<0.05$; Tables 1,2 , and 3$)$.

\subsection{Effects of UV-B and LPS on Erythrocyte Nuclear Abnormalities}

Erythrocytes of tadpoles are large cells, rounded to elliptic shape, with centric nucleus (Fig. 3A). We found nuclear abnormalities in animals from the UV and UV + LPS experiments, such as bud-shaped nuclei (Fig. 3B), anucleated cells (Fig. 3C), and micronuclei (Fig. 3D).

Exposure to UV increased nuclear abnormalities in peripheral blood erythrocytes of both pigmented and non-pigmented animals beginning in $6 \mathrm{~h}$ and continuing through all exposure times $(P<0.05)$. However, the frequency of nuclear abnormalities in non-pigmented animals is about three times greater than in pigmented ones (Fig. 3E). We found no nuclear abnormalities in the experiment manipulating LPS (Fig. 3F). However, there was an increase in nuclear abnormalities after $12 \mathrm{~h}$ in both pigmented and non-pigmented animals when animals are exposed to the combined treatment of UV + LPS, with a higher frequency in non-pigmented ones $(P<0.05$; Fig. 3G).

\subsection{Effects of UV-B and LPS on Cutaneous and Internal Melanin Pigmentation}

We found only changes in the skin pigmentation of pigmented animals after $6 \mathrm{~h}$ of UV exposition $(P<0.05$; Fig. 4). The treatment with
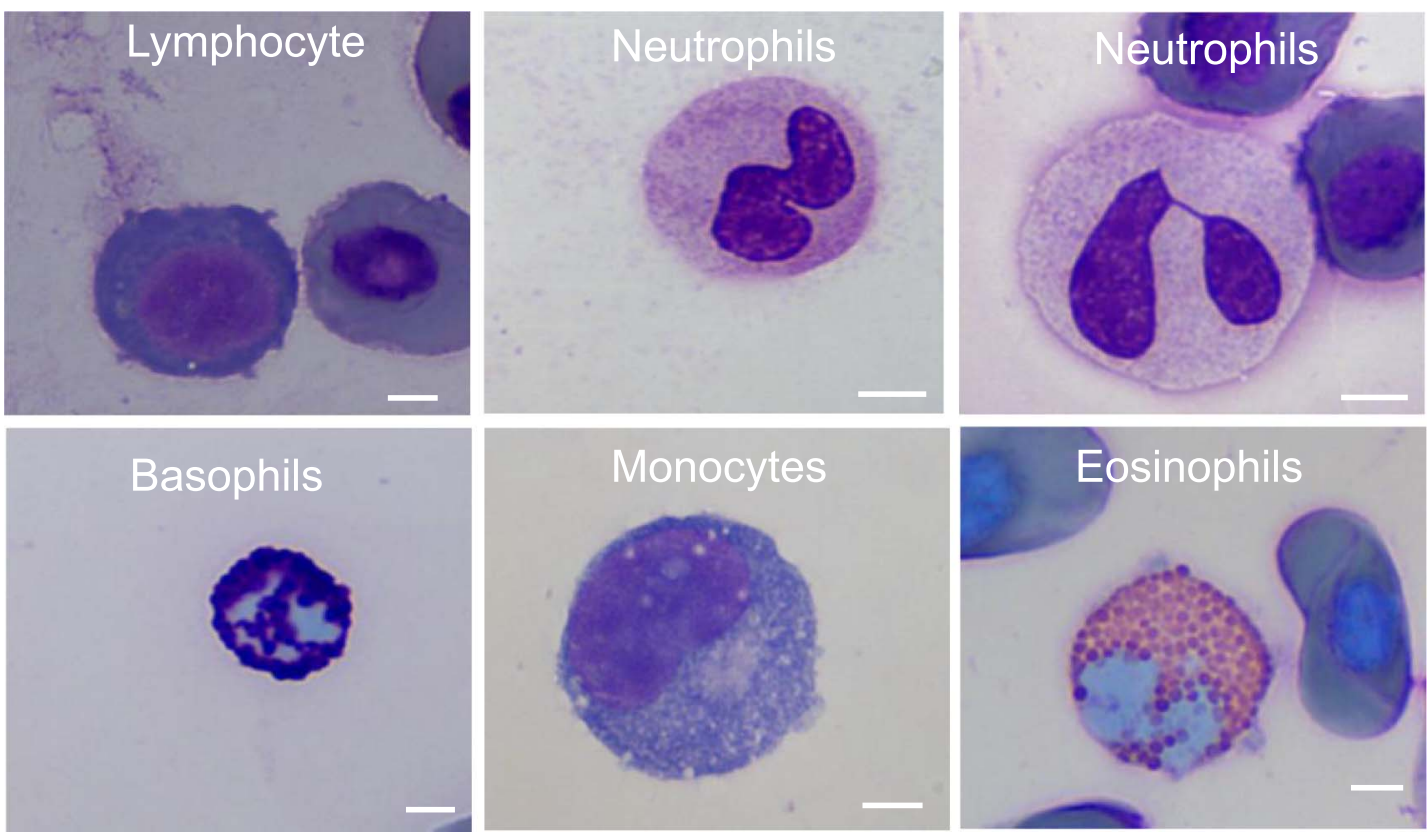

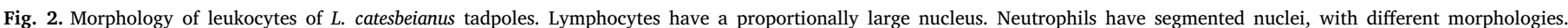

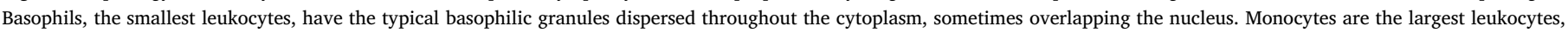
with a large nucleus. Eosinophils have numerous and typical eusinophilic granules in the cytoplasm and an eccentric nucleus. Coloration: Giemsa $7.5 \%$. Barrs: $5 \mu$ m. 
Table 1

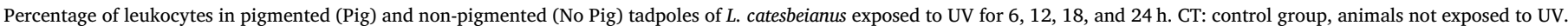

\begin{tabular}{|c|c|c|c|c|c|c|}
\hline \multicolumn{7}{|l|}{ UV } \\
\hline & & Lymphocyte & Neutrophil & Eosinophil & Monocyte & Basophil \\
\hline \multirow[t]{5}{*}{ Pig } & CT & $78.6 \pm 2.4$ & $18.2 \pm 1.6$ & $1.8 \pm 0.8$ & $0.0 \pm 0.0$ & $1.4 \pm 0.2$ \\
\hline & $6 \mathrm{~h}$ & $77.4 \pm 5.5$ & $14.2 \pm 4.5$ & $1.4 \pm 0.5$ & $0.4 \pm 0.01$ & $4.8 \pm 2.2^{*}$ \\
\hline & $12 \mathrm{~h}$ & $77.8 \pm 7.3$ & $18.8 \pm 6.7$ & $1.8 \pm 0.7$ & $0.2 \pm 0.02$ & $1.4 \pm 0.6$ \\
\hline & $18 \mathrm{~h}$ & $62.6 \pm 6.6$ & $23.6 \pm 5.9$ & $3.4 \pm 2.6$ & $4.6 \pm 2.5^{*}$ & $5.8 \pm 2.3^{*}$ \\
\hline & $24 \mathrm{~h}$ & $55.4 \pm 7.6^{*}$ & $42.2 \pm 5.2^{*}$ & $2.4 \pm 0.9$ & $1.2 \pm 0.9^{*}$ & $0.6 \pm 0.06$ \\
\hline \multirow[t]{5}{*}{ No Pig } & $\mathrm{CT}$ & $70.0 \pm 1.7$ & $12.6 \pm 3.9$ & $15.8 \pm 1.3$ & $0.4 \pm 0.24$ & $1.2 \pm 0.12$ \\
\hline & $6 \mathrm{~h}$ & $62.0 \pm 1.0$ & $13.3 \pm 3.3$ & $11.0 \pm 1.0$ & $2.5 \pm 0.9^{*}$ & $1.8 \pm 0.7$ \\
\hline & $12 \mathrm{~h}$ & $54.0 \pm 6.7^{*}$ & $9.2 \pm 3.2$ & $23.2 \pm 5.3$ & $0.6 \pm 0.2$ & $13.0 \pm 3.2^{*}$ \\
\hline & $18 \mathrm{~h}$ & $64.2 \pm 7.2$ & $17.2 \pm 9.3$ & $9.6 \pm 2.8$ & $8.4 \pm 3.0^{*}$ & $0.6 \pm 0.1$ \\
\hline & $24 \mathrm{~h}$ & $60.0 \pm 5.4$ & $14.0 \pm 1.7$ & $8.5 \pm 6.2$ & $18.3 \pm 3.2^{*}$ & $0.0 \pm 0.0$ \\
\hline
\end{tabular}

Significant differences with respect to control values are shown as *: $P<0.05$.

Table 2

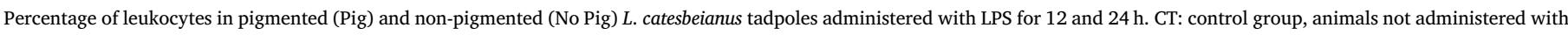
LPS.

\begin{tabular}{|c|c|c|c|c|c|c|}
\hline LPS & & & & & & \\
\hline & & Lymphocyte & Neutrophil & Eosinophil & Monocyte & Basophil \\
\hline \multirow[t]{3}{*}{ Pig } & CT & $78.6 \pm 2.4$ & $18.2 \pm 1.6$ & $1.8 \pm 0.8$ & $0.0 \pm 0.0$ & $1.4 \pm 0.2$ \\
\hline & $12 \mathrm{~h}$ & $48.2 \pm 8.0^{*}$ & $48.0 \pm 8.4^{*}$ & $2.0 \pm 0.9$ & $1.6 \pm 0.5$ & $0.0 \pm 0.0$ \\
\hline & $24 \mathrm{~h}$ & $46.0 \pm 1.3^{*}$ & $45.2 \pm 1.1^{*}$ & $1.8 \pm 0.5$ & $22.0 \pm 1.0$ & $6.8 \pm 3.2^{*}$ \\
\hline \multirow[t]{3}{*}{ No Pig } & CT & $70.0 \pm 1.7$ & $12.6 \pm 3.9$ & $15.8 \pm 1.3$ & $0.4 \pm 0.24$ & $1.2 \pm 1.2$ \\
\hline & $12 \mathrm{~h}$ & $49.6 \pm 5.8^{*}$ & $18.0 \pm 4.4$ & $15.2 \pm 3.9$ & $15.6 \pm 3.3^{*}$ & $1.6 \pm 0.1$ \\
\hline & $24 \mathrm{~h}$ & $62.8 \pm 9.0$ & $10.4 \pm 4.2$ & $17.2 \pm 7.3$ & $2.2 \pm 0.6$ & $7.4 \pm 0.4 *$ \\
\hline
\end{tabular}

Significant differences with respect to control values are shown as *: $P<0.05$.

Table 3

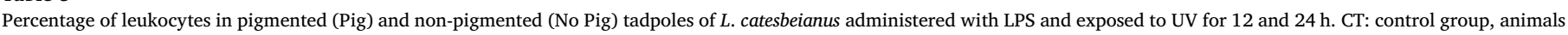
not administered with LPS nor UV exposure. Notice that non-pigmented animals did not survive after $12 \mathrm{~h}$.

\begin{tabular}{|c|c|c|c|c|c|c|}
\hline \multicolumn{7}{|c|}{ UV + LPS } \\
\hline & & Lymphocyte & Neutrophil & Eosinophil & Monocyte & Basophil \\
\hline \multirow[t]{3}{*}{ Pig } & $\mathrm{CT}$ & $78.6 \pm 2.4$ & $18.2 \pm 1.6$ & $1.8 \pm 0.8$ & $0.0 \pm 0.0$ & $1.4 \pm 0.2$ \\
\hline & $12 \mathrm{~h}$ & $68.8 \pm 6.2$ & $16.4 \pm 7.5$ & $4.8 \pm 2.6^{*}$ & $7.6 \pm 1.5$ & $2.4 \pm 0.1$ \\
\hline & $24 \mathrm{~h}$ & $48.5 \pm 1.2^{*}$ & $25.0 \pm 3.3$ & $6.5 \pm 3.1^{*}$ & $14.3 \pm 1.7$ & $5.6 \pm 0.6^{*}$ \\
\hline \multirow[t]{2}{*}{ No Pig } & CT & $70.0 \pm 1.7$ & $12.6 \pm 3.9$ & $15.8 \pm 1.3$ & $0.4 \pm 0.24$ & $1.2 \pm 1.2$ \\
\hline & $12 \mathrm{~h}$ & $54.2 \pm 2.1^{*}$ & $8.0 \pm 1.6$ & $13.0 \pm 1.8$ & $23.8 \pm 2.5^{*}$ & $1.2 \pm 0.4$ \\
\hline
\end{tabular}

Significant differences with respect to control values are shown as *: $P<0.05$.

LPS did not change body coloration $(P=0.25)$. However, tadpoles administered with LPS and exposed to UV became darker after $12 \mathrm{~h}$, and kept this phenotype after $24 \mathrm{~h}(P<0.0001$; Fig. 4I,J). The body color of non-pigment tadpoles did not change.

The heart $(G=25.08$, DF $=6 ; P=0.012)$, lungs $(G=48.85$, $\mathrm{DF}=6 ; P<0.0001)$, kidneys $(G=39.41, \mathrm{DF}=6 ; P<0.0001)$, mesenterium $(G=59.69$, DF $=6 ; P<0.0001)$, and parietal peritoneum $(G=39.93, \mathrm{DF}=6 ; P<0.0001)$ became darker after $6 \mathrm{~h}$ of $\mathrm{UV}$ exposure (Fig. 5A). The same organs became darker after $12 \mathrm{~h}$ of LPS administration (e.g., heart $=G=25.34, \quad \mathrm{DF}=6 ; \quad P<0.0001$; lungs $=G=34.46, \quad \mathrm{DF}=6 ; \quad P<0.0001 ; \quad$ kidney $=G=18.79$, $\mathrm{DF}=6 ; P=0.0047 ;$ mesenterium $=G=16.20, \mathrm{DF}=6 ; \quad P=0.012$; parietal peritoneum $=G=23.64, \mathrm{DF}=6 ; P<0.0001$ ). All organs of tadpoles in the treatment with combined UV and LPS became darker $(P<0.05$; Fig. 5C).

\subsection{Joint Effects of UV and LPS}

Body darkness is linearly and positively correlated with peritoneum ( $r=0.62 ; P=0.015)$ and kidney coloration $(r=0.93 ; P=0.03)$, as well as nuclear erythrocytes abnormalities $(r=0.83$; $P=0.02)$. The skin coloration is not correlated with immune cells. The internal coloration of the peritoneum was negatively correlated with the number of lymphocytes $(r=-0.53 ; P=0.002)$, but positively correlated with the number of monocytes $(r=0.85 ; P=0.001)$ after $24 \mathrm{~h}$ of treatment. The internal pigmentation was related to neither nuclear erythrocyte abnormalities, nor other immune cells.

\section{Discussion}

UV-B caused immunological and genotoxic effects in cells of tadpoles of $L$. catesbeianus, the latter being greater in non-pigmented animals. In addition, UV exposure increased cutaneous and internal melanin pigmentation. Melanin is a first barrier against UV effects, present in skin melanocytes and responsible for absorbing UV radiation and dissipating it in the form of heat [20].

Both pigmented and non-pigmented tadpoles had different leukocyte profiles in same developmental stage (e.g., Gosner 38-40). Pigmented animals had higher amounts of lymphocytes and neutrophils, whereas non-pigmented ones had higher amounts of eosinophils. However, pigmented tadpoles at Gosner stage 25 had lower amounts of lymphocytes and neutrophils, whereas pigmented tadpoles 

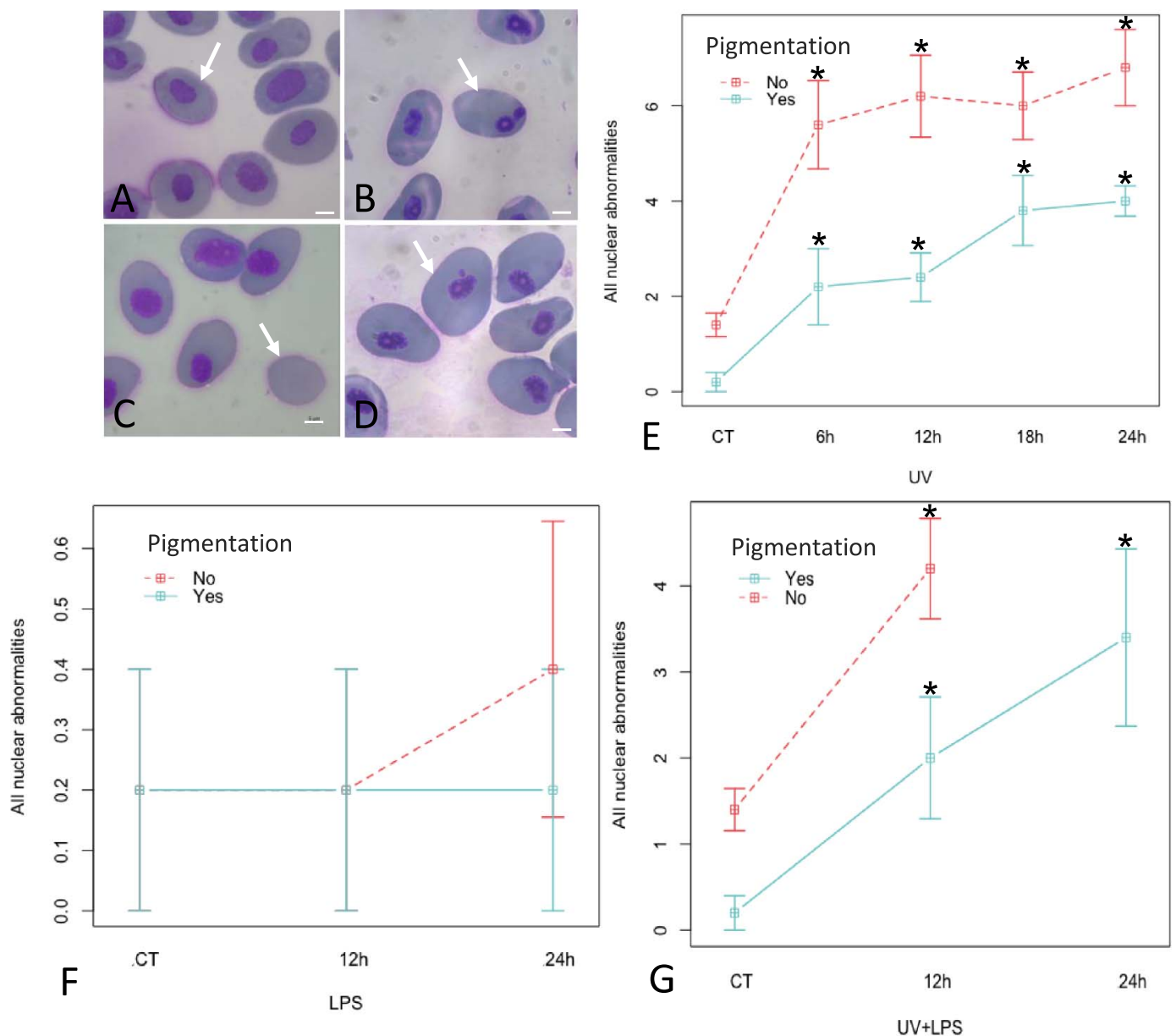

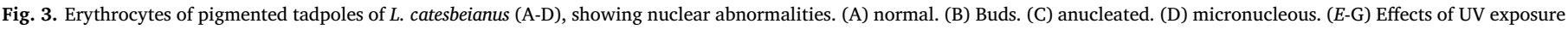

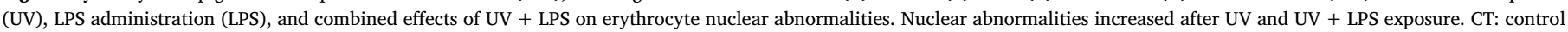

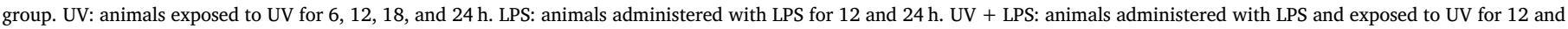
24 h. ${ }^{*}$ ) shows differences between exposure time and the control group for the same treatment. Coloration: Giemsa $7.5 \%$. Barrs: $5 \mu$ m.

at Gosner stage 38-40 had lower amounts of monocytes, basophils and eosinophils [36]. These differences in leukocyte profiles may be related to ontogeny, since the density of lymphocytes is higher throughout all developmental stages, while neutrophils are high until Gosner 40 stage and decrease afterwards up, and eosinophils and monocytes increase at the metamorphosis climax (i.e. Gosner 42; [37]). Here, we found a different leukocyte profile for tadpoles either with or without pigments at stages 38-40, which might be related to the presence of both internal and cutaneous melanin. This molecule is involved in innate immune responses in ectotherms [22] and may influence the occurrence of immune cells. Several ecotoxicological studies found that melanin plays a key role in the maintenance of animal health [19].

Leukocyte count differed in all treatments in both pigmented and non-pigmented tadpoles in late development (e.g., Gosner 38-40). Other studies found no immediate effects of UV-B on leukocytes abundance in pigmented tadpoles of Limnodynastes peronii at early development stage (e.g., Gosner 21-25; [5]). However, leukocyte amount in metamorphs exposed to UV-B during larval stages was lower than metamorphs not exposed to UV-B [5]. The same authors reported that the cumulative effect of UV does not affect directly the larval immune system, but when animals develop the adult immune system [5]. Hematological analyzes that include differential leukocyte counts provide information about individual's immune status and aid in presumptive or definitive diagnosis [38]. Lymphocytes decreased in all treatments in both pigmented and non-pigmented tadpoles. Lymphocytes are important in specific immune responses and in the production of growth factors for blood cells [39,40]. The hypothalamic-pituitary-interrenal axis (HPI) is activated in anurans under environmental stresses, and lymphocytes are lost and other leukocytes are mobilized after corticosteroid release [41]. Basophils increased in all experiments likely because they have rapid hypersensitivity to different treatments. Monocytes increased to large counts in all experiments only in nonpigmented animals. Monocytes play key roles in bacterial infections [42] and were expected to increase in animals administered with LPS. A previous study [11] found similar pattern in basophils and monocytes of Sclerophrys regularis exposed to UV-A. In this scenaria, we expect that melanin could act in the innate immune defense of pigmented animals, but monocytes are specific immune cells and do not act in this context.

Hepatic mast cells increased in all experiments. Mast cells contribute to immune responses and modulate inflammation [15]. UV activates skin mast cells and induces release of histamine [43] and increases skin mast cells after 3-4 h [44]. LPS also increases mast cells in the connective tissue of mammals [15]. Here, we demonstrated for the first time that UV also affects mast cells in non-irradiated organs of tadpoles. Thus, the increase in these cells in our experiments suggests systemic effects of UV radiation in tadpoles and could indicate that the tissue is inflamed.

Exposure to UV induced nuclear abnormalities in the erythrocytes of both pigmented and non-pigmented animals. UV radiation damages the DNA. The frequency of nuclear abnormalities increases in adult anurans (e.g., Physalaemus nattereri) after $18 \mathrm{~h}$ of UV exposure [2]. Here, we found a strong increase in nuclear abnormalities after $6 \mathrm{~h}$ in tadpoles 

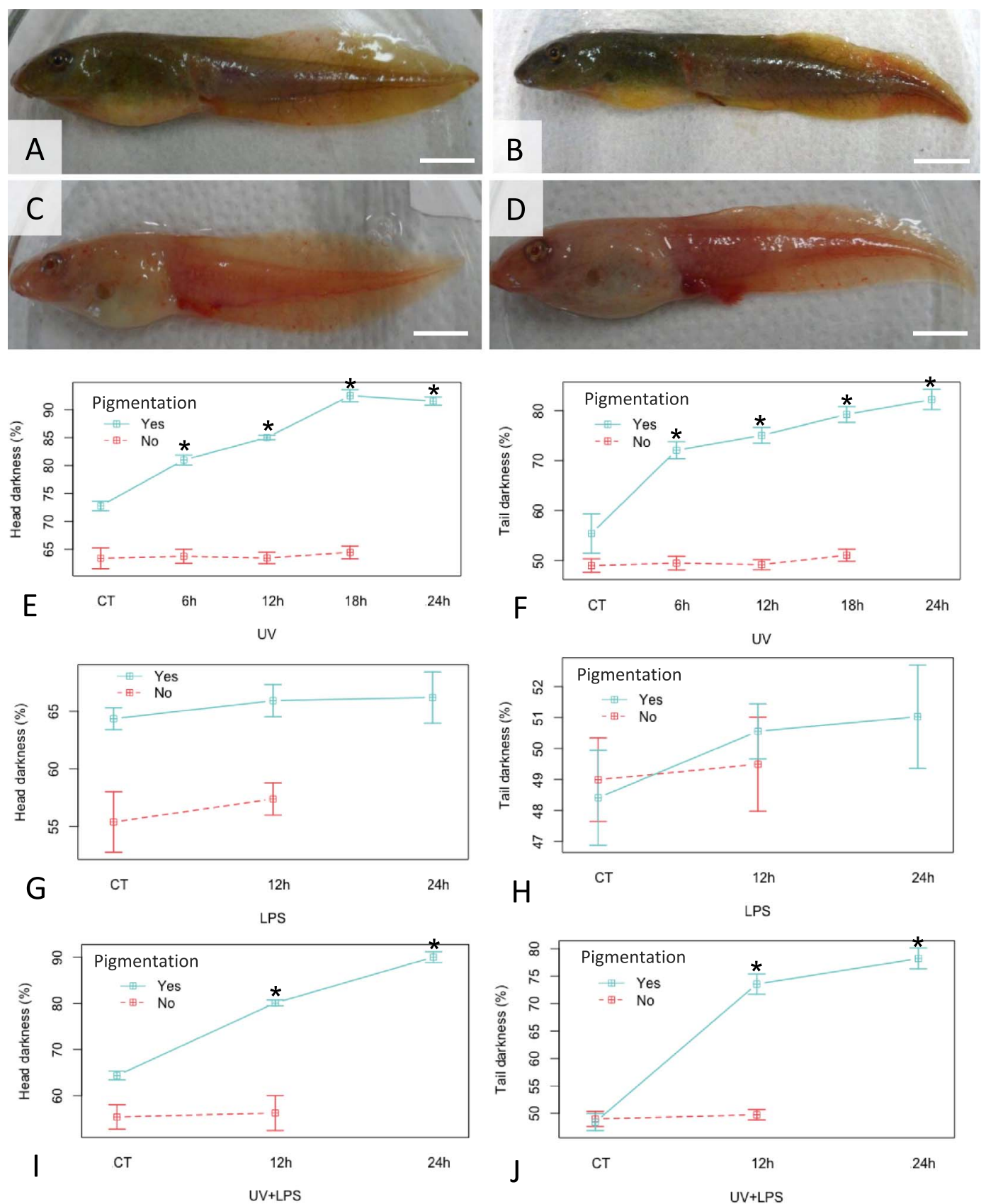

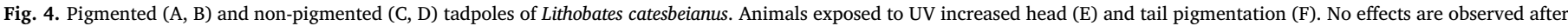

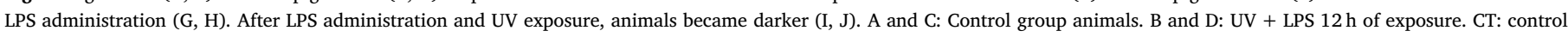

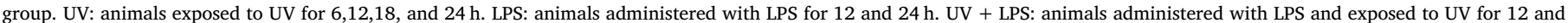
24 h. (*) shows differences between exposure time and the control group in the same treatment. Barrs: $0.8 \mathrm{~cm}$.

exposed to UV. These distinct patterns of nuclear abnormalities between adults of $P$. nattereri and larvae of $L$. catesbeianus are similar to that observed for genotoxicity in fish, in which the genetic material of individuals at early developmental stages are more sensitive to damage [45]. Here, we found that non-pigmented tadpoles were more prone to genotoxic effects than pigmented ones. In pigmented animals, melanin provides a protective barrier to UV that can absorb the radiation and dissipate it as heat and presence of melanin became the pigmented animals more resistant.

Pigmented tadpoles became darker only when exposed to UV. Exposure to UV-B radiation for short periods increases skin pigmentation by dispersion of the melanin present within the melanocytes [46]. Conversely, prolonged exposure is usually associated with deleterious effects, such as skin cancer [46]. The darkening of the tadpole body here was positively correlated with nuclear abnormalities, demonstrating that the rapid increase in cutaneous pigmentation confers a first barrier against UV effects. However, if the effect is prolonged, there may be irreversible genotoxic damage [2].

The body darkness of tadpoles exposed to UV was correlated with internal melanin in the peritoneum, demonstrating that UV affects pigmentation beyond the skin. Internal pigmentation was inversely correlated with lymphocytes after $24 \mathrm{~h}$. This seems to be a compensatory response, since lymphocytes are involved with specific immune responses $[39,40]$ and melanin is involved in the innate immune response [22]. Peritoneal melanin was also linearly correlated with monocytes in all treatments, demonstrating that they have complementary functions in the response against pathogens, in which monocytes act in inflammation [42] and melanin with its bactericide 

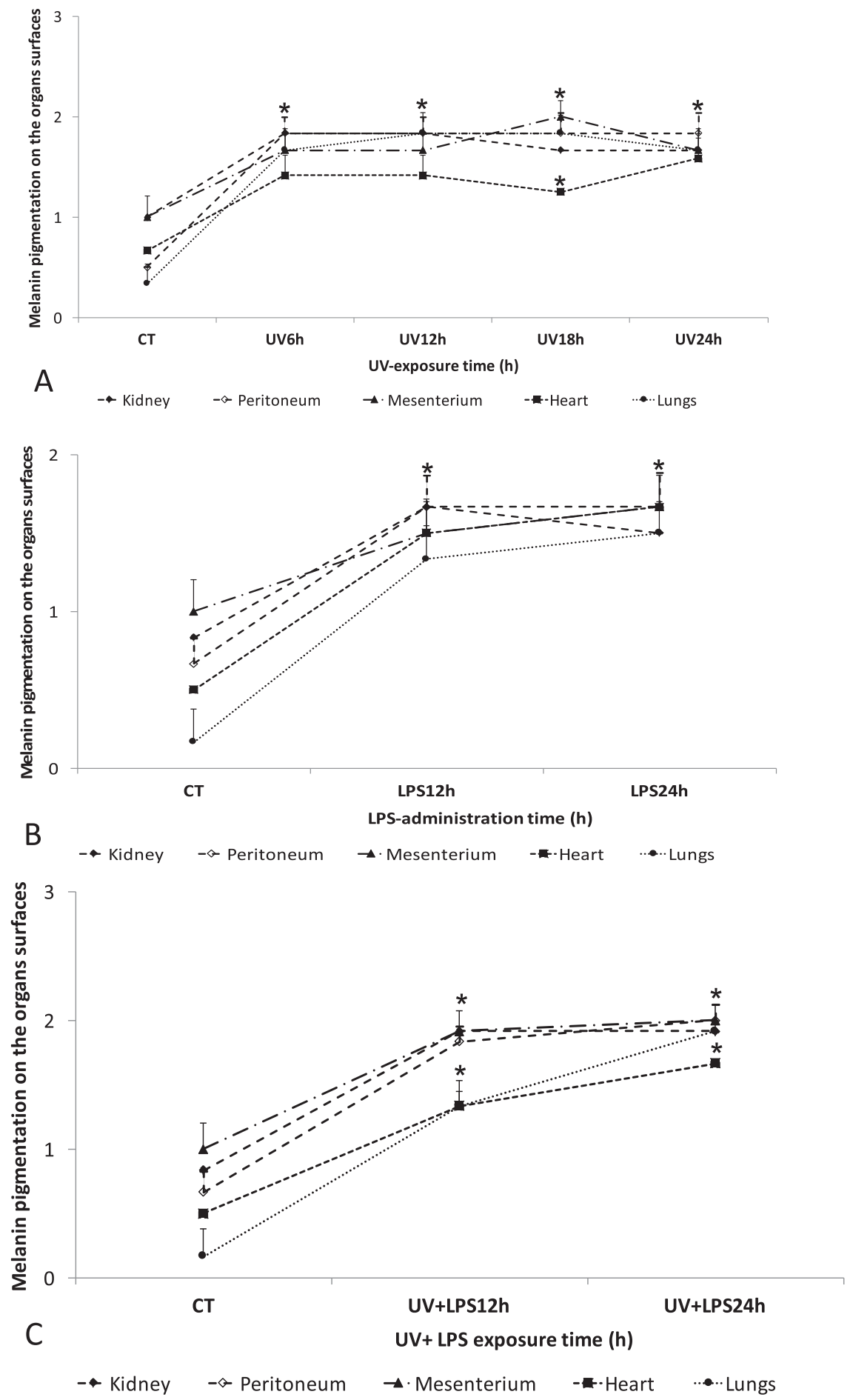

Fig. 5. Visceral melanin pigmentation responses of tadpoles of Lithobates catesbeianus after UV (A), LPS (B) and UV + LPS (C) treatment. CT: control group. UV: animals exposed to UV for 6,12,18, and $24 \mathrm{~h}$. LPS: animals administered with LPS for 12 and $24 \mathrm{~h}$. UV + LPS: animals administered with LPS and exposed to UV for 12 and $24 \mathrm{~h}$. ( $\left.^{*}\right)$ shows differences between exposure time and the control group in the same treatment. function [22]. Therefore, internal and cutaneous pigmented cells seem to have distinct physiological responses.

\section{Conclusion}

Exposure to UV affected immune cells of both non-pigmented and pigmented tadpoles, but genotoxic effects were more severe in nonpigmented ones. Both UV and LPS induced hepatic inflammation, as demonstrated by mast cell analysis. Leukocyte responses are faster in non-pigmented animals, supporting the idea that melanin is involved in the initial innate immune response. The hypothesis that internal melanin protects against UV is corroborated by the higher genotoxicity in non-pigmented L. catesbeianus tadpoles. Further studies are necessary to understand what mechanisms melanin plays in this protection.

\section{Acknowledgments}

Gabriela B. Leite and Bruno S.L. Valverde helped during experiments. Diogo B. Provete and Juan M. Pérez Iglesias helped with data analyses. Diogo B. Provete kindly reviewed the English language. LFB received a post-doctoral fellowship from FAPESP (2014/00946-4). This project was supported by a grant from FAPESP to CO (2015/12006-9). LFB received a post-doctoral fellowship from CAPES (PNPD) during the final preparation of this manuscript. LZF received a doctoral fellowship 
from FAPESP (2017/07971-2). CO received a fellowship from CNPq $(305081 / 2015-2)$.

\section{References}

[1] A.R. Blaustein, L.K. Belden, Amphibian defenses against ultraviolet-B radiation, Evol. Dev. 5 (2003) 89-97.

[2] L. Franco-Belussi, H.N. Sköld, C. de Oliveira, Internal pigment cells respond to external UV radiation in frogs, J. Exp. Biol. 219 (2016) 1378-1383.

[3] J. Hearst, The structure of photolyase: using photon energy for DNA repair, Science 268 (5219) (1995) 1858.

[4] L.A. Rollins-Smith, J.P. Ramsey, J.D. Pask, L.K. Reinert, D.C. Woodhams, Amphibian immune defenses against Chytridiomycosis: impacts of changing environment, Integr. Comp. Biol. (2011) 1-11.

[5] E. Ceccato, R.L. Cramp, F. Seebacher, C.E. Franklin, Early exposure to ultraviolet-B radiation decreases immune function later in life, Conserv. Physiol. 4 (2016) cow037.

[6] J.M. Kiesecker, A.R. Blaustein, Synergism between UV-B radiation and a pathogen magnifies amphibian embryo mortality in nature, Proc. Natl. Acad. Sci. 92 (24) (1995) 11049-11052.

[7] L.A. Alton, C.E. Franklin, Drivers of amphibian declines: effects of ultraviolet radiation and interactions with other environmental factors, Clim. Chang. Responses 4 (1) (2017) 6.

[8] A.K. Davis, D.L. Maney, J.C. Maerz, The use of leukocyte profiles to measure stress in vertebrates: a review for ecologists, Funct. Ecol. 22 (2008) 760-772.

[9] A.M. Attademo, P.M. Peltzer, R.C. Lajmanovich, M.C. Cabagna-Zenklusen, C.M. Junges, A. Basso, Biological endpoints, enzyme activities, and blood cell parameters in two anuran tadpole species in rice agroecosystems of mid-eastern Argentina, Environ. Monit. Assess. 186 (2014) 635-649.

[10] M.F. Medina, M.E. González, S.M.R. Klyver, I.M.A. Odstrcil, Histopathological and biochemical changes in the liver, kidney, and blood of amphibians intoxicated with cadmium, Turk. J. Biol. 40 (2016) 229-238.

[11] A.E.D.H. Sayed, Hematotoxic and biochemical effects of UVA on the Egyptian toad (Bufo regularis), Int. J. Radiat. Biol. 92 (2016) 35-41.

[12] Z.M. Zhelev, G.S. Popgeorgiev, M.V. Angelov, Investigating the changes in the morphological content of the blood of Pelophylax ridibundus (Amphibia: Ranidae) as a result of anthropogenic pollution and its use as an environmental bioindicator, Acta Zool. Bulg. 65 (2013) 87-196.

[13] C.F. Benjamim, J.S. Silva, Z.B. Fortes, M.A. Oliveira, S.H. Ferreira, F.Q. Cunha, Inhibition of leukocyte rolling by nitric oxide during sepsis leads to reduced migration of active microbicidal neutrophils, Infect. Immun. 70 (2002) 3602-3620.

[14] B. Shakoory, S.M. Fitzgerald, S.A. Lee, D.S. Chi, G. Krishnaswamy, The role of human mast cell-derived cytokines in eosinophil biology, J. Interf. Cytokine Res. 24 (2004) 271-281.

[15] P.S. da Silva, A.P. Girol, S.M. Oliani, Mast cells modulate the inflammatory process in endotoxin-induced uveitis, Mol. Vis. 17 (2011) 1310-1319.

[16] C. Flores Quintana, F. Ruas de Moraes, Respuesta Inflmatoria a la Inoculación de LPS em Pacu (Piaractus mesopotamicus) Suplementados com Cromo, Rev. Ictiol. 9 (2001) 13-19.

[17] S. Akira, H. Hemmi, Recognition of pathogen-associated molecular patterns by TLR family, Immunol. Lett. 85 (2003) 85-95.

[18] C.D. Baroni, L. Ruco, G.S. de Franceschi, S. Uccini, L. Adorini, G.D. Cnen, Biological effects of lipoposaccharide (LPS) in vivo, Immunology 31 (1976) 217-224.

[19] C. De Oliveira, L. Franco-Belussi, L.Z. Fanali, L.R.S. Santos, Use of melanin-pigmented cells as a new tool to evaluate effects of agrochemicals and other emerging contaminants in Brazilian anurans, Ecotoxicology and Genotoxicology: Non-traditional Terrestrial Models, 32 2017, p. 125.

[20] W. Montagna, K. Carlisle, The architecture of black and white facial skin, J. Am. Acad. Dermatol. 24 (1991) 929-937.

[21] J.P. Ortonne, Photoprotective properties of skin melanin, Br. J. Dermatol. 146 (2002) 7-10.

[22] R.E. Wolke, C.J. George, V.S. Blazer, Pigmented Macrophages Accumulations (MMC; PMB): Possible Monitors of Fish Health, in: W.G. HargisJr. (Ed.), Parasitology and Pathology of Marine Organisms of the World Ocean, 1985, pp. 93-97.
[23] V.O. van Uitregt, R.S. Wilson, C.E. Franklin, Cooler temperatures increase sensitivity to ultraviolet B radiation in embryos and larvae of the frog Limnodynastes Peronii, Glob. Chang. Biol. 13 (2007) 1114-1121.

[24] K.L. Gosner, A simplified table for staging anuran embryos and larvae with notes on identification, Herpetologica 16 (1960) 183-190.

[25] A.P. Schuch, M.B. dos Santos, V.M. Lipinski, L.V. Peres, C.P. dos Santos, S.Z. Cechin, N.J. Schuch, D.K. Pinheiro, E.L. da Silva Loreto, Identification of influential events concerning the Antarctic ozone hole over southern Brazil and the biological effects induced by UVB and UVA radiation in an endemic treefrog species, Ecotoxicol. Environ. Saf. 118 (2015) 190-198.

[26] S.H. Shirley, E.A. Grimm, D.F. Kusewitt, Ultraviolet radiation and the slug transcription factor induce Proinflammatory and immunomodulatory mediator expression in melanocytes, J. Skin Cancer (2012), http://dx.doi.org/10.1155/2012/ 410925.

[27] Y. Chen, P. Hao, A. Dang, Optimal transform in perceptually uniform color space and its application in image coding, Image Anal. Recognit. (2004) 269-276.

[28] P.A. Svensson, E. Forsgren, T. Amundsen, H. Sköld, Chromatic interaction between egg pigmentation and skin chromatophores in the nuptial coloration of female twospotted gobies, J. Exp. Biol. 208 (2005) 4391-4397.

[29] R Core Team, R: A Language and Environment for Statistical Computing, R Foundation for Statistical Computing, Vienna, Austria, 2017.

[30] D. Lüdecke, sjPlot: data visualization for statistics in social science, R Package Version 2.1.2, 2016 https://CRAN.R-project.org $/$ package $=$ sjPlot.

[31] M.J. Crawley, The R Book, John Wiley \& Sons, London, 2012.

[32] E.L. Moen, C.J. Fricano-Kugler, B.W. Luikart, A.J. O'Malley, Analyzing clustered data: why and how to account for multiple observations nested within a study participant? PLoS One 11 (2016) e0146721.

[33] D.A. Fournier, H.J. Skaug, J. Ancheta, J. Ianelli, A. Magnusson, M.N. Maunder, A. Nielsen, J. Sibert, AD model builder: using automatic differentiation for statistical inference of highly parameterized complex nonlinear models, Optim. Methods Softw. 27 (2012) 233-249.

[34] R.V. Lenth, Least-squares means: the R package lsmeans, J. Stat. Softw. 69 (2016) $1-33$.

[35] F. Cribari-Neto, A. Zeileis, Beta Regression in R, J. Stat. Softw. 34 (2010) 1-24.

[36] C.S. Carvalho, H.S.M. Utsunomiya, T. Pasquoto, R. Lima, M.J. Costa, M.N. Fernandes, Blood cell responses and metallothionein in the liver, kidney and muscles of bullfrog tadpoles, Lithobates catesbeianus, following exposure to different metals, Environ. Pollut. 221 (2017) 445-452.

[37] A.K. Davis, Metamorphosis-related changes in leukocyte profiles of larval bullfrogs (Rana catesbeiana), Comp. Clin. Pathol. 18 (2009) 181-186.

[38] S.H. Newman, J.F. Piatt, J. White, Hematological and plasma biochemical reference ranges of Alaskan seabirds: their ecological significance and clinical importance, Colon. Waterbirds (1997) 492-504.

[39] H. Arikan, K. CiCek, Haematology of amphibians and reptiles: a review. North-west, J. Zool. 10 (2014) 190-209.

[40] J.A. Claver, A.I.E. Quaglia, Comparative morphology, development, and function of blood cells in nonmammalian vertebrates, J. Exot. Pet Med. 18 (2009) 87-97.

[41] L.A. Rollins-Smith, Amphibian immunity-stress, disease, and climate change, Dev. Comp. Immunol. 66 (2017) 111-119.

[42] T.W. Campbell, C.K. Ellis, Hematology ofamphibians, Avian and Exotic Animal Hematology and Cytology, Blackwell Publishing, Ames (IA), 2007, pp. 83-91.

[43] H. Siiskonen, A. Smorodchenko, K. Krause, M. Maurer, Ultraviolet radiation and skin mast cells: effects, mechanisms, and relevance for skin diseases, Exp. Dermatol. 27 (2018) 3-8.

[44] P.H. Hart, M.A. Grimbaldeston, J.J. Finlay-Jones, Mast cells in UV-B-induced immunosuppression, J. Photochem. Photobiol. B Biol. 55 (2000) 81-87.

[45] I. Domingues, R. Oliveira, J. Lourenço, C.K. Grisolia, S. Mendo, A.M.V.M. Soares, Biomarkers as a tool to assess effects of chromium (VI): comparison of responses in zebrafish early life stages and adults, Comp. Biochem. Physiol. Part C Toxicol. Pharmacol. 152 (2010) 338-345.

[46] N. Agar, A.R. Young, Melanogenesis: a photoprotective response to DNA damage? Mutat. Res. 571 (2005) 121-132.

[47] L. Franco-Belussi, R. Zieri, L.R. De Souza Santos, R.M. Moresco, C. De Oliveira, Pigmentation in anuran testes: anatomical pattern and variation, Anat. Rec. 292 (2) (2009) 178-182. 